\title{
Validade da citologia cervicovaginal na deteç̧ão de lesões pré-neoplásicas e neoplásicas de colo de útero
}

Validity of cervicovaginal cytology for detection of cancerous and precancerous lesions of the cervix

\author{
Adriana de Araujo Pinho \\ Maria Cristina F. Iwama de Mattos ${ }^{2}$
}

\begin{tabular}{|c|c|}
\hline & \\
\hline $\begin{array}{l}\text { Câncer cervical } \\
\text { Teste de Papanicolaou } \\
\text { Validade } \\
\text { Diagnóstico } \\
\text { Sensibilidade } \\
\text { Especificidade }\end{array}$ & $\begin{array}{l}\text { Introdução: O exame colpocitológico ou teste de Papanicolaou permite o diagnóstico precoce } \\
\text { do câncer de colo do útero. O êxito no rastreamento desta doença dependerá, além de outros } \\
\text { fatores, da acuidade diagnóstica do exame colpocitológico. Objetivos: Avaliar a acurácia } \\
\text { diagnóstica da citologia cervicovaginal na detecção de lesões cervicais através da comparação } \\
\text { citoistopatológica. Material e métodos: Avaliou-se o grau de concordância entre os exames } \\
\text { citológico e histopatológico de } 373 \text { pacientes atendidas em um hospital universitário no } \\
\text { período de } 1990 \text { a } 1997 \text { e foram calculados os indicadores: sensibilidade, especificidade, } \\
\text { valores preditivos positivos e negativos e as taxas de falsos positivos e falsos negativos do } \\
\text { exame. Resultados: A taxa bruta de concordância citoistopatológica foi de } 65,1 \% \text {. A sensibilida- } \\
\text { de do exame foi alta (96,0\%), no entanto sua especificidade foi baixa ( } 51,5 \%) \text {, significando a } \\
\text { inclusão de muitos resultados falsos positivos. A taxa de falsos positivos foi de } 48,4 \% \text {, enquanto } \\
\text { a de falsos negativos foi de } 4 \% \text {. Discussão: Apesar de a sensibilidade do teste ser alta, sua } \\
\text { especificidade é baixa, significando que muitas mulheres seriam falsamente diagnosticadas } \\
\text { como doentes, levando a um número elevado de resultados falsos positivos e custos desneces- } \\
\text { sários, além do potencial caráter iatrogênico que esta ação poderia assumir. No entanto, um } \\
\text { exame altamente sensível é o teste de escolha para programas de rastreamento de câncer } \\
\text { cervical na população feminina. Conclusões: Para garantir a acuidade diagnóstica do teste de } \\
\text { Papanicolaou são essenciais atividades de controle de qualidade nos laboratórios, tanto do } \\
\text { procedimento colpocitológico quanto do histopatológico, permitindo, assim, o êxito na } \\
\text { deteccão precoce e no tratamento das lesões cervicais. }\end{array}$ \\
\hline
\end{tabular}

Background: The cervicovaginal cytology or Papanicolaou (Pap) test allows the early diagnosis of cervical cancer. Success of cervical cancer screening depends, among other factors, on the diagnostic accuracy of Pap test. Objectives: To evaluate the diagnostic accuracy of cervicovaginal cytology in the detection of cervical lesions through cito-histopathological correlation. Methods: We evaluated the degree of correlation between the cytological results and cervical biopsies from 373 patients assisted at a university hospital in the period from 1990 through 1997. The indicators: sensitivity, specificity, positive and negative predictive values and false positive and false negative rates for cervicovaginal cytology diagnosis were calculated. Results: The crude rate of cito-histopathological correlation was $65.1 \%$. The sensitivity of the Pap test was high (96\%); its specificity, however, was low (51.5\%), resulting in the inclusion of many false positive results. The rate of false positive results

was $48.4 \%$, whereas the rate of false negative results was 4\%. Discussion: Despite the higher sensitivity of the test, its specificity is low, meaning that a lot of women would be falsely diagnosed as ill, resulting in a high number of false positive results and unnecessary costs, in addition to the potential iatrogenic character of this preventive practice. However, a highly sensitive test is the test of choice for the screening of cervical cancer among the female population. Conclusions: In order to guarantee the diagnostic accuracy of the Pap test, it is imperative to establish quality control activities for colpocytological and histopathological procedures, thus allowing success in early detection and treatment of cervical lesions. key words

Cervical cancer

Papanicolaou test

Validity

Diagnosis

Sensitivity

Specificity
1. Mestre em Saúde Pública da Faculdade de Saúde Pública Universidade de São Paulo 2. Docente da Faculdade de Medicina de Botucatu Universidade Estadual Paulista. Trabalho realizado no Departamento de Patologia da Faculdade de Medicina de Botucatu da Universidade Estadual Paulista com auxilio financeiro da Fundação de Amparo à Pesquisa do Estado de São Paulo (Fapesp) (Processo 98/04402-4). Apresentado no VI Congresso Paulista de Saúde Pública Aguas de Lindóia, de 17 a 20 de outubro de 1999. 


\section{Introdução}

No município de Botucatu, o câncer de colo do útero é uma das causas mais freqüentes de mortalidade feminina. Dados de registro hospitalar revelaram que, no Hospital de Clínicas da Faculdade de Medicina de Botucatu, este tipo de câncer é a segunda neoplasia maligna mais freqüente em mulheres (4). Trata-se, portanto, de um importante problema para a saúde da mulher.

O câncer de colo uterino é uma doença cuja evolução é lenta, apresentando fases pré-invasivas e, portanto, benignas, caracterizadas por lesões conhecidas como neoplasias intra-epiteliais cervicais. O período de evolução de uma lesão cervical inicial para a forma invasiva e, por conseguinte, maligna é de aproximadamente 20 anos (7). Este período relativamente longo permite que ações preventivas sejam eficientes e alterem o quadro epidemiológico da doença. Estas ações se fazem por meio da educação popular, detecção e diagnóstico precoces e tratamento das lesões cervicais precursoras. Agindo precocemente, pode-se alterar a história natural da doença, proporcionando a diminuição de sua morbidade e mortalidade.

O diagnóstico precoce do câncer do colo uterino permite o rastreamento, ou screening, das lesões de colo em suas fases iniciais antes de se tornarem lesões invasivas através de um método de detecção conhecido como colpocitologia oncológica ou exame de Papanicolaou.

O êxito no rastreamento do câncer de colo uterino e de suas lesões precursoras dependerá, além de outros fatores, da acuidade diagnóstica do exame colpocitológico, ou seja, de sua precisão em diagnosticar corretamente os casos verdadeiros de lesões cervicais neoplásicas e préneoplásicas (sensibilidade) e aqueles casos que não apresentam qualquer tipo de alteração epitelial (especificidade).

O exame de Papanicolaou tem sido considerado um método altamente confiável para detectar as lesões cervicais. A sensibilidade do teste, ou seja, a proporção de casos verdadeiros positivos detectados pelo exame de Papanicolaou já foi descrita como sendo próxima de $99,8 \%$ (6). Soost et al. (13) encontraram uma sensibilidade de $79,9 \%$, e a especificidade, ou seja, a proporção de casos verdadeiros negativos detectados pelo teste, foi de $99,9 \%$. Além de sua acuidade diagnóstica, o exame de Papanicolaou é considerado um método de baixo custo, simples e de fácil execução. Estas características o tornam um método amplamente utilizado em programas de controle do câncer cervicouterino.
Algumas medidas podem ser utilizadas para melhorar a qualidade do exame colpocitológico e, portanto, aumentar sua acuidade diagnóstica. Uma delas é medir o erro do teste, ou seja, determinar a taxa de falsos negativos e falsos positivos detectados pelo teste de Papanicolaou. Essas taxas são importantes indicadores de acuidade diagnóstica do exame colpocitológico, além da sensibilidade e especificidade, e podem ser determinadas através da correlação citoistopatológica (12), ou seja, comparando-se os resultados dos exames citológicos com os resultados dos exames histopatológicos (e vice-versa), observando, assim, se há concordância entre estes dois métodos. Vários trabalhos têm utilizado a correlação citoistopatológica como uma forma de avaliar a acuidade diagnóstica do exame de Papanicolaou $(1,8$, $9,10,14)$.

Além disso, a concordância dos métodos utilizados no diagnóstico das lesões cervicais é importante na conduta terapêutica utilizada no combate à neoplasia cervical intraepitelial, além de fatores intrínsecos à paciente (3), pois evita o tratamento cirúrgico para os casos iniciais de neoplasia cervical diagnosticados pela colpocitologia antes de confirmá-los por outros métodos, observando assim se há concordância entre eles para então prosseguir ou não o tratamento.

Logo, a colpocitologia, apesar de não ser dada como diagnóstico final, é de grande importância junto aos demais métodos no rastreamento do câncer cervicouterino e de suas lesões precursoras, como também no auxílio à orientação do tratamento a ser seguido. Sendo assim, torna-se necessário avaliar a acurácia diagnóstica da citologia cervicovaginal na detecção de lesões pré-neoplásicas e neoplásicas do colo uterino.

\section{Material e métodos}

Foram selecionados, inicialmente, 680 exames colpocitológicos positivos de 100.899 exames realizados no Hospital de Clínicas do município de Botucatu, no período de 1990 a 1997.

O teste padrão utilizado para avaliar a acuidade diagnóstica do exame colpocitológico é o exame histopatológico de uma amostra de tecido obtida através de biópsia cervical. Para comparar os exames colpocitológicos e histopatológicos, seus respectivos diagnósticos foram padronizados segundo a classificação de Bethesda (1988). 
A fim de se proceder à correlação citoistopatológica, foram consideradas somente as pacientes que apresentavam dados referentes à colpocitologia e à histopatologia, num total de 373 , conforme os seguintes critérios: 1 ) intervalo entre o exame citológico e o histopatológico confirmativo dentro de um ano; 2) pacientes com mais de um exame colpocitológico (somente o primeiro exame foi considerado para a análise, desde que a paciente apresentasse o exame histopatológico realizado até um ano após a colpocitologia).

Avaliou-se o grau de concordância entre os exames colpocitológicos e histopatológicos, calculando-se o coeficiente kappa, como também foram calculados os indicadores: sensibilidade, especificidade, valores preditivos positivo e negativo e as taxas de falsos positivos e de falsos negativos do exame colpocitológico.

\section{Cálculo dos indicadores}

\section{Sensibilidade}

É a capacidade que um teste apresenta de detectar os indivíduos verdadeiramente doentes. Este indicador é calculado através da fórmula:

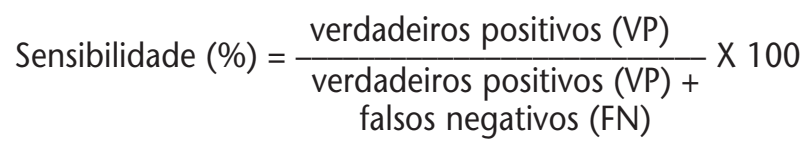

No presente estudo, foi definido como teste verdadeiro positivo a presença dos diagnósticos de: células escamosas atípicas de significado indeterminado (Ascus); lesões intra-epiteliais escamosas de baixo grau (LSIL), as quais incluem os diagnósticos de alterações celulares consistentes com infecção pelo papiloma vírus humano (HPV) e displasia leve ou neoplasia intra-epitelial cervical de grau l; lesões intra-epiteliais cervicais de alto grau (HSIL), que incluem os diagnósticos de displasia moderada ou neoplasia intra-epitelial cervical de grau II e displasia grave ou neoplasia intra-epitelial cervical de grau III ou carcinoma in situ; carcinoma invasor e adenocarcinoma confirmados pela histopatologia.

Os exames citológicos de pacientes reconhecidas histopatologicamente com a lesão cervical que não diagnosticaram anormalidades no epitélio cervical foram considerados falsos negativos.

\section{Especificidade}

É a capacidade que o teste apresenta de detectar os indivíduos verdadeiramente sadios. Este indicador é calculado através da fórmula:

Especificidade $(\%)=\frac{\text { verdadeiros negativos }(\mathrm{VN})}{\text { falsos positivos }(\mathrm{FP})+} \times 100$

Para o cálculo da especificidade, considerou-se verdadeiro negativo a ausência de qualquer alteração epitelial, confirmado pela histopatologia. Os exames citológicos de pacientes reconhecidas histopatologicamente sem a lesão cervical que diagnosticaram anormalidades no epitélio cervical foram considerados falsos positivos.

\section{Valor preditivo positivo}

É a probabilidade de ter a doença em um paciente com resultado positivo do teste. É calculado a partir da fórmula:

$\begin{gathered}\text { Valor preditivo } \\ \text { positivo (\%) }\end{gathered}=\frac{\begin{array}{c}\text { verdadeiros positivos (VP) } \\ \text { verdadeiros positivos (VP) } \\ \text { falsos positivos (FP) }\end{array}}{\text { fal }} \times 100$

\section{Valor preditivo negativo}

É a probabilidade de não ter a doença quando o resultado do teste é negativo. É calculado a partir da fórmula:

Valor preditivo $=\frac{\text { verdadeiros negativos }(\mathrm{VN})}{\text { negativo }(\%)}=100$

Para o cálculo das taxas de falsos positivos e de falsos negativos utilizaram-se as equações apresentadas por Renshaw (12), que define:

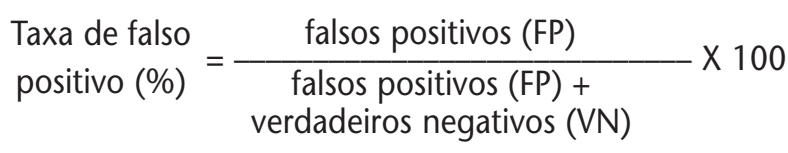

$$
\begin{aligned}
& \text { Taxa de falso } \\
& \text { negativo (\%) }
\end{aligned}=\frac{\text { falsos negativos (FN) }}{\text { falsos negativos (FN) }+} \times 100
$$




\section{Resultados}

A taxa bruta de concordância citoistopatológica foi de $65,1 \%$. Os resultados foram mais concordantes para as lesões mais graves, como nos casos de adenocarcinoma $(85,7 \%)$ e nos de carcinoma invasor $(82,3 \%)$; e menos concordantes para os diagnósticos de HSIL $(67,2 \%)$ e LSIL (48,9\%) (Tabela 1). A concordância citoistopatológica foi boa, com valor kappa de 0,64.

Para o cálculo de sensibilidade, especificidade e valores preditivos do exame colpocitológico, considerou-se como critério para delimitar resultados positivos daqueles negativos a presença dos diagnósticos de Ascus, LSIL, HSIL, carcinoma invasor e adenocarcinoma. Utilizando-se este ponto de corte, a sensibilidade do teste foi alta $(96 \%)$. No entanto, a especificidade foi baixa $(51,5 \%)$ (Tabela 2). Isto significa que, na população estudada, a chance de serem detectados os indivíduos verdadeiramente doentes é de $96 \%$. O resultado do teste será raramente negativo na presença da doença, ou seja, dá poucos resultados falsos negativos. A taxa observada de falsos negativos foi baixa (4\%).

A especificidade do teste nesse mesmo ponto de corte foi muito baixa, o que acarretou a inclusão de muitos exames falsos positivos. A taxa de falsos positivos foi de $48,4 \%$. $\mathrm{O}$ teste não diagnosticou corretamente os casos negativos nessa população.

A sensibilidade também foi calculada para os diferentes graus de lesão cervical, utilizando-se este mesmo pon- to de corte para delimitar os resultados positivos. A sensibilidade da colpocitologia foi maior para os diagnósticos de HSIL, carcinoma invasor e adenocarcinoma (100\%) do que para o diagnóstico de LSIL, cuja sensibilidade foi de 85,3\% (Tabela 3).

Os valores preditivos positivos e negativos encontrados na população estudada foram altos, $85 \%$ e $82 \%$, respectivamente, quando o critério utilizado para estabelecer um resultado positivo foi a presença de qualquer alteração epitelial. Isto significa que $85 \%$ dos diagnosticados como positivos são de pessoas verdadeiramente doentes e que $82 \%$ dos diagnosticados como negativos correspondem a pacientes verdadeiramente sadios.

\section{Discussão}

A correlação citoistopatológica mostrou que a sensibilidade do teste de Papanicolaou é muito alta, considerando teste positivo a presença de qualquer alteração no epitélio cervical. No entanto, sua especificidade é baixa. Isto significa que, ao se utilizar este ponto de corte para definir as pacientes com resultados positivos, praticamente todas as pessoas doentes seriam detectadas, pois o teste foi muito sensível, mas muitas pessoas seriam diagnosticadas falsamente como doentes. A taxa de falsos positivos foi relativamente alta $(48,4 \%)$. Isto levaria muitas mulheres com diagnósticos suspeitos a prosseguirem em outros exames, mais invasivos, acarretando custos

\section{Tabela 1 de Clínicas no periodo de 1990 a 1997}

\begin{tabular}{|c|c|c|c|c|c|c|c|c|c|c|c|c|}
\hline \multicolumn{13}{|c|}{ Diagnóstico histopatológico } \\
\hline \multirow{2}{*}{$\begin{array}{l}\text { Exame } \\
\text { citológico }\end{array}$} & \multicolumn{2}{|c|}{ Negativo } & \multicolumn{2}{|c|}{ LSIL } & \multicolumn{2}{|c|}{ HSIL } & \multicolumn{2}{|c|}{ Ca invasor } & \multicolumn{2}{|c|}{ Adenocarcinoma } & \multicolumn{2}{|c|}{ Total } \\
\hline & $n$ & $\%$ & $n$ & $\%$ & $n$ & $\%$ & $n$ & $\%$ & $n$ & $\%$ & $n$ & $\%$ \\
\hline Negativo & $50^{*}$ & 82 & 11 & 18 & 0 & - & 0 & - & 0 & - & 61 & 16,4 \\
\hline Ascus & 5 & 33,3 & 2 & 13,3 & 2 & 33,4 & 5 & 33,3 & 1 & 6,7 & 15 & 4 \\
\hline LSIL & 23 & 24,5 & $46^{*}$ & 48,9 & 24 & 25,5 & 1 & 1,1 & 0 & - & 94 & 25,2 \\
\hline HSIL & 16 & 12 & 16 & 11,9 & $90^{*}$ & 67,2 & 11 & 8,2 & 1 & 0,7 & 134 & 35,9 \\
\hline Ca invasor & 3 & 4,8 & 0 & - & 7 & 11,3 & $51^{*}$ & 82,3 & 1 & 1,6 & 62 & 16,6 \\
\hline Adenocarcinoma & 0 & - & 0 & - & 1 & 14,3 & 0 & - & 6* & 85,7 & 7 & 1,9 \\
\hline Total & 97 & 26 & 75 & 20,1 & 124 & 33,3 & 68 & 18,2 & 9 & 2,4 & 373 & 100 \\
\hline
\end{tabular}

*Concordância citoistopatológica.

Ascus: células escamosas atípicas de significado indeterminado; LSIL: lesões intra-epiteliais escamosas de baixo grau; HSIL: lesões intra-epiteliais escamosas de alto grau; Ca invasor: carcinoma invasor. 
Sensibilidade, especificidade e valores preditivos do exame

Tabela 2 colpocitológico

\begin{tabular}{lccc} 
Exame & \multicolumn{2}{c}{ Diagnóstico histopatológico } & Total \\
\cline { 2 - 3 } citológico & Positivo & Negativo & \\
Positivo & $265^{*}$ & 47 & 312 \\
Negativo & 11 & 50 & 61 \\
Total & 276 & 97 & 373
\end{tabular}

*Resultados diagnosticados como Ascus, LSIL, HSIL, Ca invasor e adenocarcinoma. Sensibilidade $265 / 276=96 \% \quad$ Valor preditivo $+265 / 312=85 \%$ Especificidade $50 / 97=51,5 \% \quad$ Valor preditivo- $50 / 61=82 \%$ Taxa de falso-positivo $47 / 97=48,4 \% \quad$ falso-negativo $11 / 276=4 \%$

\section{Sensibilidade do exame} colpocitológico para os diferentes Tabela 3 graus de lesão cervical

\begin{tabular}{lcccc} 
Exame & \multicolumn{4}{c}{ Diagnóstico histopatológico } \\
\cline { 2 - 5 } citológico & LSIL & HSIL & Ca inv. & Adenocarc. \\
Negativo & 11 & 0 & 0 & 0 \\
$\begin{array}{l}\text { Ascus/LSIL/ } \\
\quad \text { HSIL/Ca inv./ }\end{array}$ & 64 & 124 & 68 & 9 \\
$\quad$ adenocarcinoma & & & & \\
Total & 75 & 124 & 68 & 9 \\
Sensibilidade (\%) & 85,3 & 100 & 100 & 100 \\
\hline
\end{tabular}

Ascus: células escamosas atípicas de significado indeterminado; LSIL: lesões intra-epiteliais escamosas de baixo grau; HSIL: lesões intra-epiteliais escamosas de alto grau; Ca inv.: carcinoma invasor.

desnecessários, além do potencial caráter iatrogênico que esta ação poderia assumir para muitas pacientes.

Além disso, o baixo valor de especificidade encontrado deve ser visto no contexto da população estudada, pois o cálculo da especificidade é determinado pelo número de casos verdadeiros negativos e falsos positivos (8). $\mathrm{Na}$ população estudada, o número de casos verdadeiros negativos foi bem menor do que os verdadeiros positivos, pois a maioria das pacientes com exames colpocitológicos negativos não tem biópsia confirmativa e por isso não está representada no presente estudo. Logo, os casos verdadeiros negativos estão sub-representados. Além do mais, devido aos resultados colpocitológicos positivos conduzirem a uma investigação mais precisa, um grande número de casos falsos positivos foi identificado.

A taxa de resultados falsos negativos encontrada foi baixa e ocorreu devido à classificação de casos de LSIL como negativos. A taxa de falsos negativos pode ser atribuída a três tipos de erro: de amostragem, no rastreamento e na interpretação do esfregaço (12). Mais da metade de todos os resultados falsos negativos é atribuída à amostragem inadequada (5). Um erro de amostragem ocorre quando as células de uma lesão não estão presentes no esfregaço cervicovaginal. Como não houve revisão citológica dos casos falsos negativos, não há como saber que tipo de erro ocorreu.

Quanto aos resultados falsos positivos, apesar de ser esperado que eles sejam quase que exclusivamente devidos ao erro de interpretação do esfregaço, alguns autores já relataram que, ao reexaminarem, presumiram que havia erro na amostragem da biópsia, pois a revisão dos esfregaços confirmou a presença de lesão cervical, indicando que a biópsia não foi representativa da lesão (8). A falta de acurácia na biópsia cervical ocorre principalmente para detectar lesões de grau mais leves (1). Mesmo durante a colposcopia a falha da biópsia em detectar a lesão pode ocorrer devido à localização das lesões em áreas de difícil amostragem, tal como no interior do canal cervical (1).

Portanto deve-se levar em consideração a qualidade do exame histopatológico, pois a comparação do exame colpocitológico com a biópsia cervical continua sendo um importante elemento de avaliação de qualidade em muitos laboratórios, onde a histologia é considerada o teste padrão para a correlação citoistopatológica (14).

Além disso, o ponto de corte estabelecido para delimitar os resultados positivos daqueles negativos terá grande influência na sensibilidade e especificidade do exame colpocitológico. Os níveis de sensibilidade e especificidade variam quando são eliminados os resultados de Ascus e LSIL e considerados somente os de HSIL, carcinoma invasor e adenocarcinoma como pontos de corte para delimitar os resultados positivos. Há uma diminuição da sensibilidade para $83,6 \%$ e um aumento da especificidade para $79,6 \%$. Esta elevação significa que nesse ponto de corte houve uma diminuição de exames falsos positivos. A taxa de falsos positivos foi mais baixa (20,3\%) quando comparada com a do ponto de corte anterior.

Alguns autores consideram resultados positivos os diagnósticos de HSIL, carcinoma invasor e adenocarcinoma, e justificam a eliminação dos diagnósticos de LSIL e Ascus pelo fato de estas lesões, geralmente, não serem confirmadas histopatologicamente, sugerindo-se, na maioria das vezes, somente o seguimento citopatológico, pois estas lesões podem regredir espontaneamente num período de seis a 18 meses (13).

Por outro lado, a diminuição da sensibilidade levou a um aumento de resultados falsos negativos, ou seja, au- 
mentaria o número de pessoas com a doença que não seriam detectadas pelo teste. A taxa de falsos negativos foi de $16,4 \%$.

Ao diminuir ainda mais o ponto de corte, considerando-se apenas os diagnósticos de carcinoma invasor e adenocarcinoma como critério para delimitar resultados positivos, a sensibilidade diminui para $75,3 \%$ e a especificidade aumenta, consideravelmente, para $96,3 \%$. Isto significa que o teste seria considerado muito específico, mas muitas pessoas com a doença não seriam detectadas utilizando-se este ponto de corte, ou seja, a taxa de falsos negativos seria maior (24,6\%). Porém o teste, neste caso, conseguiria excluir praticamente todas as mulheres que realmente não tinham a doença. A taxa de falsos positivos foi muito pequena $(3,7 \%)$.

Como foi apresentado, o fato de variar o ponto de corte para o rastreamento das lesões cervicais fez mudar os níveis de sensibilidade e especificidade do teste. Isso mostra como é difícil contrabalançar estes dois indicadores e que ambos, raramente, serão de $100 \%$, o que seria considerar perfeito o exame colpocitológico. Entretanto, como o propósito do teste é detectar precocemente as lesões pré-neoplásicas e neoplásicas do colo uterino, utilizando, deste modo, como critério para teste positivo, a presença de qualquer alteração no epitélio cervical, a sensibilidade encontrada (96\%) foi muito boa. Um exame altamente sensível deve ser o teste de escolha num programa de rastreamento do câncer cervical na população feminina, em que se desejam detectar todos os casos positivos de lesões cervicais.

Os valores preditivos do teste foram calculados a partir dos resultados presentes na Tabela 2, onde os casos positivos foram definidos como qualquer tipo de alteração epitelial. No entanto, estes valores referem-se somente à população estudada $(n=373)$, ou seja, as pacientes atendidas no Hospital de Clínicas de Botucatu, cujo exame colpocitológico havia sido confirmado histopatologicamente, segundo o critério estabelecido.

A sensibilidade e a especificidade do teste são propriedades exclusivas do teste diagnóstico e não variam consideravelmente, a não ser por mudanças na técnica ou por erros na sua aplicação (11), ao passo que os valores preditivos dependem, além destes indicadores, da prevalência da doença na população que está sendo testada, ou seja, do número de casos positivos existentes na população total definida em um determinado ponto do tempo.

Segundo Fletcher et al. (2), "resultados positivos, mesmo de um teste muito específico aplicado em populações de baixa prevalência da doença poderão ser, em grande parte, falsos positivos. O mesmo acontece em populações com alta prevalência da doença, em que resultados negativos de um teste, mesmo sendo considerado de alta sensibilidade, poderão ser falsos negativos quando aplicados em uma população com grandes chances de ter a doença".

A população estudada não é representativa da população de Botucatu e região, por constituir uma amostra do Hospital de Clínicas de Botucatu, para onde são encaminhados os casos mais graves da doença. Desta forma, a prevalência da doença é alta e não corresponde à verdadeira prevalência da doença na população à qual o teste deverá ser aplicado na prática (2). A prevalência de resultados positivos obtida pela aplicação do teste em uma determinada população, principalmente numa população selecionada como a do presente estudo, não é sinônimo de prevalência da doença na região onde foi feito o estudo (11).

Esse resultado não invalida o uso do valor preditivo como indicador de qualidade do exame colpocitológico, porém ele deve ser analisado dentro do contexto da população estudada, tendo-se em vista a prevalência da doença na mesma.

\section{Conclusões}

Os indicadores usados na validação do exame colpocitológico são influenciados, como foi visto, por vários fatores que dizem respeito tanto ao procedimento citológico quanto ao histopatológico. O controle de qualidade destes dois procedimentos, como também a avaliação de qualidade nos laboratórios através da correlação citoistopatológica, são essenciais para garantir a sensibilidade e a especificidade do exame de Papanicolaou.

No entanto, para garantir o êxito na detecção precoce e no tratamento das lesões cervicais, outros fatores são importantes, além da necessária acuidade diagnóstica do exame colpocitológico, como a cobertura do teste de Papanicolaou entre a população feminina; a proporção de mulheres rastreadas em cada faixa etária, principalmente a de maior risco do ponto de vista epidemiológico; a periodicidade adequada na realização do exame colpocitológico; a eficiência do diagnóstico e um adequado seguimento terapêutico das mulheres para eliminar as lesões cervicais.

Acima de tudo, o sucesso no rastreamento e controle do câncer de colo uterino dependerá da reorganização da assis- 
tência clinicoginecológica às mulheres nos serviços de saúde; da capacitação dos profissionais de saúde; do aumento dos recursos humanos e financeiros destinados a esta área $\mathrm{e}$ da implementação efetiva de políticas públicas que resgatem os princípios de universalidade e integralidade presentes no Programa de Atenção Integral à Saúde da Mulher.

\section{Referências}

I. DiBonito, L. Cervical cytopathology: an evaluation of its accuracy based on cytohistologic comparison. Cancer, 72: 3002-6, 1993.

2. Fletcher, R.H. et al. Epidemiologia clínica. Porto Alegre: Artes Médicas, |99|.

3. Focchi, J. et al. Lesões precursoras do câncer do colo do útero: diagnóstico e tratamento.J. Bras. Ginecol., 97:299-303, 1987.

4. Fundação Oncocentro de São Paulo (FOSP). Registro hospitalar de câncer. São Paulo: A Fundação, 1996.

5. Gill, G.W. Pap smear risk management by process control. Cancer Cytopath., 81 (4): 198-21 I, 1997.

6. Haam, E.A comparative study of the accuracy of cancer cell detection by cytological methods. Acta Cytol., 6(6): 508-I I, 1962.

7. Halbe, H.W. Câncer de colo do uterino: conceito, importância, incidência e fatores de risco. In:Tratado de ginecologia. São Paulo: Roca, 1993. v. 2.

8. Jones, B. A. \& Novis, D. A. Cervical biopsy-cytologic correlation: a college of American pathologists Q-Probes study of
22439 correlations in 348 laboratories. Arch. Pathol. Lab. Med., 120 (6): 523-31, 1996.

9. Kainz, C. et al. Influence of age and human papillomavirus: infection on reliability of cervical cytopathology. Arch. Gynecol. Obstet., 256: 23-8, 1995.

10. Oliveira, L.J. et al. Correlação colposcópica, citopatológica e histológica do câncer do colo uterino. Rev. Bras. Ginecol Obstet., II (8): I50-2, 1989.

I।. Pereira, M. G. Aferição dos eventos. In: Epidemiologia: teoria e prática. Rio de Janeiro: Guanabara Koogan, 1995. cap. 17.

12. Renshaw,A.A. Analysis of error in calculating the false-negative rate in the interpretation of cervicovaginal smears. Cancer Cytopathol., 8I (5): 264-7I, 1997.

13. Soost, H. et al. The validation of cervical cytology: sensitivity, specificity and predictive values. Acta Cytol., 35( I): 8- 13, I99 I.

14. Tritz, D.M. et al. Etiologies for non-correlating cervical cytologies and biopsies. Am.J. Clin.Pathol., 103(5): 594-7, 1995. 